Pacific

Journal of

Mathematics

ON THE CLASSIFICATION OF FINITE GROUPS ACTING ON HOMOLOGY 3-SPHERES

BRUNO ZimMERMANN

Volume $217 \quad$ No. 2

December 2004 


\title{
ON THE CLASSIFICATION OF FINITE GROUPS ACTING ON HOMOLOGY 3-SPHERES
}

\author{
BRUNO ZimMERMANN
}

\begin{abstract}
In previous work we showed that the only finite nonabelian simple group acting by diffeomorphisms on a homology 3sphere is the alternating or dodecahedral group $\mathbb{A}_{5}$. Here we characterize finite nonsolvable groups that act on a homology 3-sphere preserving orientation. We find exactly the finite nonsolvable groups that act orthogonally on the 3-sphere, plus two families of groups for which we do not know at present if they really can act on a homology 3-sphere.
\end{abstract}

\section{Introduction}

A finite group acting freely on a homology $n$-sphere has periodic cohomology of period $n+1$, and the groups with periodic cohomology have been characterized by Zassenhaus and Suzuki (see [AM, Theorem 6.15] or [W, Chapter 6.3]). Specializing to dimension three one gets a list of the possible finite groups which may act freely on a homology 3 -sphere (see [Mn]). The complete classification of such groups remains open (the difficulty lies in the class of groups $Q(8 n, k, l)$ defined below).

Here we are interested in (not necessarily free) actions of finite groups on homology 3 -spheres. Such groups no longer have periodic cohomology. It has been shown in $[\mathbf{Z 2}]$ that the only finite nonabelian simple group acting on a homology 3 -sphere is the alternating group $\mathbb{A}_{5} \cong \operatorname{PSL}(2,5)$. Continuing work begun in $[\mathbf{R}]$ (see also $[\mathbf{Z 1}]$ ), the main result of the present paper is a characterization of the finite nonsolvable groups which act on homology 3spheres. We find exactly the finite nonsolvable groups admitting orthogonal actions on the 3-sphere (subgroups of $\mathrm{SO}(4)$ ), plus two additional classes of groups for which we cannot decide at present if they can really act on a homology 3 -sphere. We remark that a similar analysis should be possible also for the case of solvable groups, but the list of such groups will be inevitably much longer and more technical.

In order to state our results we introduce some notation.

By $[\mathbf{M n}]$, any finite group acting freely on a homology $n$-sphere has at most one involution, which consequently belongs to the center of the group; in the following, we denote by $Z \cong \mathbb{Z}_{2}$ the subgroup generated by such an 
involution. If $G_{1}$ and $G_{2}$ are two such groups, we denote by

$$
G_{1} \times{ }_{Z} G_{2}
$$

the central product of $G_{1}$ and $G_{2}$, which is the product of the two groups with the two central involutions identified (the quotient of the product by the order-two subgroup generated by (involution of $G_{1}$, involution of $G_{2}$ ); see [S1, p. 137]). Thus $G_{1}$ and $G_{2}$ commute elementwise and $G_{1} \cap G_{2}=Z$.

We denote by $\mathbb{D}_{4 n}^{*}, \mathbb{A}_{4}^{*}, \mathbb{S}_{4}^{*}$ and $\mathbb{A}_{5}^{*}$ the binary dihedral, tetrahedral, octahedral and dodecahedral group (of orders 4n, 24, 48 and 120, respectively). These are the preimages under the surjection of Lie groups $S^{3} \rightarrow \mathrm{SO}(3)$ of the dihedral group $\mathbb{D}_{2 n}$, the tetrahedral group $\mathbb{A}_{4}$, the octahedral group $\mathbb{S}_{4}$ and the dodecahedral group $\mathbb{A}_{5}$. Together with the cyclic groups $\mathbb{Z}_{n}$, these are exactly the finite subgroups of the orthogonal group $\mathrm{SO}(3)$.

Following $[\mathbf{M n}]$, for relatively coprime positive integers $8 n, k$ and $l$, let $Q(8 n, k, l)$ denote the group with presentation

$$
\left\langle x, y, z \mid x^{2}=(x y)^{2}=y^{2 n}, z^{k l}=1, x z x^{-1}=z^{r}, y z y^{-1}=z^{-1}\right\rangle,
$$

where $r \equiv-1 \bmod k$ and $r \equiv+1 \bmod l$. Then $Q(8 n, k, l)$ is an extension with normal subgroup $\mathbb{Z}_{k} \times \mathbb{Z}_{l} \cong \mathbb{Z}_{k l}$ and factor group the binary dihedral group $\mathbb{D}_{8 n}^{*} \cong Q(8 n, 1,1)$. Among the groups $Q(8 n, k, l)$ are the only candidates of finite groups not admitting free orthogonal actions on the 3 -sphere but possibly admitting nonorthogonal free actions. Some of these groups act freely on homology 3-spheres, but it is not known if any of them can act on $S^{3}$ (see Section 2).

Theorem. Let $G$ be a finite nonsolvable group of orientation-preserving diffeomorphisms of a homology 3-sphere. Then $G$ is isomorphic to one of the following groups:

(i) $\mathbb{A}_{5}$ or $\mathbb{A}_{5} \times \mathbb{Z}_{2}$.

(ii) $\mathbb{A}_{5}^{*} \times_{Z} \mathbb{A}_{5}^{*}, \mathbb{A}_{5}^{*} \times_{Z} \mathbb{S}_{4}^{*}, \mathbb{A}_{5}^{*} \times_{Z} \mathbb{A}_{4}^{*}, \mathbb{A}_{5}^{*} \times_{Z} \mathbb{D}_{4 n}^{*}$ or $\mathbb{A}_{5}^{*} \times_{Z} \mathbb{Z}_{2 n}$.

(iii) $\mathbb{A}_{5}^{*} \times_{Z} Q(8 n, k, l)$, for relatively coprime integers $8 n, k$ and $l$, with $n$ odd and $n>k>l \geq 1$.

(iv) $\mathbb{A}_{5}^{*} \times_{Z}\left(\mathbb{D}_{4 n}^{*} \times \mathbb{Z}_{k}\right)$, with $n$ odd and $k>1$ coprime to $4 n$.

In case (i), each involution of $\mathbb{A}_{5}$ has nonempty connected fixed-point set and in all other cases, each factor of the central products acts freely.

The groups of type (i) and (ii) are exactly the finite nonsolvable groups that admit orientation-preserving orthogonal actions on the 3-sphere.

Considering $S^{3}$ as the group of unit quaternions, we have the surjection of Lie groups $S^{3} \times S^{3} \rightarrow \mathrm{SO}(4)$ induced by left and right multiplication of $S^{3}$. The kernel of this surjection is the group $Z$ of order two generated by $(-1,-1)$, so $\mathrm{SO}(4)$ is isomorphic to the central product $S^{3} \times_{Z} S^{3}$. The finite subgroups of $S^{3}$ are exactly the cyclic groups $\mathbb{Z}_{n}$ and the binary polyhedral groups $\mathbb{D}_{4 n}^{*}, \mathbb{A}_{4}^{*}, \mathbb{S}_{4}^{*}$ and $\mathbb{A}_{5}^{*}$. Thus all groups of type (ii) in the theorem act 
orthogonally on the 3 -sphere. (See [DV] for a list of all finite groups acting orthogonally on $S^{3}$.)

The group $\mathbb{A}_{5}^{*} \times_{Z} \mathbb{A}_{5}^{*}$ occurs as the symmetry group of the 4-dimensional regular 120-cell whose boundary is the 3 -sphere with a regular tessellation by 120 regular spherical dodecahedra with dihedral angles $2 \pi / 3$. The characteristic cell (simplex) of the regular spherical $2 \pi / 3$-dodecahedron is the Coxeter tetrahedron of type $[3,3,5]$, so $\mathbb{A}_{5}^{*} \times_{Z} \mathbb{A}_{5}^{*}$ is the orientation-preserving subgroup of index two in the corresponding Coxeter group (generated by the reflections in the faces of the tetrahedron). Similarly, an action of $\mathbb{A}_{5}$ on $S^{3}$ comes from the orientation-preserving symmetry group of the regular 4-simplex, which induces a regular tessellation of $S^{3}$ by five regular $2 \pi / 4$ tetrahedra having the Coxeter tetrahedron of type $[3,3,3]$ as their characteristic cell. There is another action of $\mathbb{A}_{5}$ on $S^{3}$, obtained by doubling the standard action of $\mathbb{A}_{5}$ on the 3-ball. See $[\mathbf{D u}]$ for a geometric description of the quotient orbifolds of the finite subgroups of $\mathrm{SO}(4)$ occurring in the theorem.

\section{Preliminaries}

Any finite group $G$ acting freely on the 3-sphere or on a homology 3-sphere has cohomological period four and at most one involution. By [Mn], the groups of period four and with at most one involution are exactly the following:

2.1. . The groups $1, \mathbb{D}_{4 n}^{*}, \mathbb{A}_{4}^{*}, \mathbb{S}_{4}^{*}$ and $\mathbb{A}_{5}^{*}$.

2.2. . The split metacyclic groups $D_{2^{k}(2 n+1)}$ with presentation

$$
\left\langle x, y \mid x^{2^{k}}=1, y^{2 n+1}=1, x y x^{-1}=y^{-1}\right\rangle,
$$

where $k \geq 2$ and $n \geq 1$. The group $D_{4(2 n+1)}$ is isomorphic to $\mathbb{D}_{4(2 n+1)}^{*}$.

2.3. . The groups $P_{8 \cdot 3^{k}}^{\prime}$ with presentation

$$
\left\langle x, y, z \mid x^{2}=(x y)^{2}=y^{2}, z x z^{-1}=y, z y z^{-1}=x y, z^{3^{k}}=1\right\rangle,
$$

where $k \geq 1$; these groups are extensions with normal subgroup the quaternion group $Q_{8}$ of order eight (generated by $x$ and $y$ ) and factor group the cyclic group of order $3^{k}$. The group $P_{24}^{\prime}$ is isomorphic to $\mathbb{A}_{4}^{*}$.

2.4. . The groups $Q(8 n, k, l)$, for relatively coprime integers $8 n, k$ and $l$ and either:

(a) $n$ odd and $n>k>l \geq 1$, or

(b) $n \geq 2$ even and $k>l \geq 1$. 
2.5. . The groups $P_{48 r}^{\prime \prime}$, with $r \geq 3$ odd, which are extensions with normal subgroup $\mathbb{Z}_{r}$ and factor group $\mathbb{S}_{4}^{*}$ such that the 3-Sylow subgroup is cyclic, and such that the commutator subgroup $\mathbb{A}_{4}^{*}$ of $\mathbb{S}_{4}^{*}$ acts trivially on $\mathbb{Z}_{r}$ and the remaining elements by -identity. See [L, p. 195] for presentations of these groups.

2.6. . The product of any of these groups with a cyclic group of relatively prime order.

The groups of types 2.1-2.3 and their products with a cyclic group of relatively prime order are exactly the groups that act orthogonally and freely on $S^{3}$. It has been shown in $[\mathbf{L}]$ that the groups of types 2.4(b) and 2.5 do not act freely on a homology 3 -sphere. The situation for the groups of type 2.4(a) is not completely understood: some of them act freely on homology 3 -spheres, some do not, and it doesn't seem to be known at present if any of them can act on the 3 -sphere (see $[\mathbf{M g}]$; see also $[\mathbf{K}$, problem 3.37 , p. 173]: in contrast to the claim in the updated version of the problem list the classification of the finite groups acting on the 3 -sphere remains open, however).

We collect some other results needed for the proof of the main theorem. For a proof of Proposition 1, see [B, Theorems 7.9 and 8.1], and [RZ, Lemma 3] for part (c).

\section{Proposition 1.}

(a) The fixed-point set of a diffeomorphism of prime period $p$ of a $\mathbb{Z}_{p^{-}}$ homology 3-sphere is either empty or connected.

(b) For a prime $p$, the group $\mathbb{Z}_{p} \times \mathbb{Z}_{p}$ does not act freely on a $\mathbb{Z}_{p}$-homology 3-sphere.

(c) For a prime $p$, let $A \cong \mathbb{Z}_{p} \times \mathbb{Z}_{p}$ be a finite group of orientationpreserving diffeomorphisms of a $\mathbb{Z}_{p}$-homology 3 -sphere. Then there are exactly two subgroups $\mathbb{Z}_{p}$ of $A$ with nonempty fixed-point sets (two disjoint circles) or, if $p=2$, all three involutions in A may have nonempty fixed-point sets which are three circles intersecting in exactly two points (so A has two global fixed points).

Proposition 2. Let $G$ be a finite group of orientation-preserving diffeomorphisms of a closed orientable 3-manifold. Suppose that $G$ contains an element $h$ with nonempty connected fixed-point set. Then the normalizer $N_{G} h$ of the subgroup generated by $h$ in $G$ is isomorphic to a subgroup of a semidirect product $\mathbb{Z}_{2} \ltimes\left(\mathbb{Z}_{a} \times \mathbb{Z}_{b}\right)$, for some nonnegative integers $a$ and $b$, where $\mathbb{Z}_{2}$ acts on the normal subgroup $\mathbb{Z}_{a} \times \mathbb{Z}_{b}$ by sending each element to its inverse.

Proof. The fixed-point set of $h$ is a simple closed curve $K$ invariant under the action of $N_{G} h$. By a result of Newman [N] (see also [Dr]), a periodic transformation of a connected manifold that is the identity on an open subset 
is the identity. Thus the action of an element of $N_{G} h$ is determined by its action in a regular neighbourhood of $K$ where it is a standard action on a solid torus. Every element of $N_{G} h$ restricts to a reflection (strong inversion) or to a (possibly trivial) rotation on $K$. The subgroup of rotations is abelian and has index one or two in $N_{G} h$. It has a cyclic subgroup (the elements acting trivially on $K$ ), with cyclic quotient group, so it is abelian of rank at most two.

\section{Proposition 3.}

(a) The only finite abelian group of rank $\geq 3$ that acts on a homology 3sphere preserving orientation is the elementary abelian 2 -group $\mathbb{Z}_{2} \times$ $\mathbb{Z}_{2} \times \mathbb{Z}_{2}$.

(b) Let $H$ be a metacyclic group, with normal subgroup $\mathbb{Z}_{p}$ and factor group $\mathbb{Z}_{q}$, for a prime number $p$ and an integer $q \geq 2$. If $H$ acts by orientation-preserving diffeomorphisms on a homology 3-sphere, any generator of $\mathbb{Z}_{q}$ acts as \pm identity on $\mathbb{Z}_{p}$.

Proof. (a) An abelian group $A$ of rank three has subgroups $\mathbb{Z}_{p} \times \mathbb{Z}_{p}$, for some prime $p \geq 2$. By Proposition 1 , some element $h$ of order $p$ has nonempty connected fixed-point set, so $A$ is a semidirect product of the type described in Proposition 2. The only abelian group of rank three of such type is the group $\mathbb{Z}_{2} \times \mathbb{Z}_{2} \times \mathbb{Z}_{2}$.

(b) Follows from [Z2, Proof of Proposition 1].

\section{Proof of the theorem}

A finite group $F$ is quasisimple if it is perfect (the abelianized group is trivial) and the factor group $F / Z(F)$ of $F$ by its center $Z(F)$ is a nonabelian simple group. A group $E$ is semisimple if it is perfect and the factor group $E / Z(E)$ is a direct product of nonabelian simple groups. A semisimple group $E$ is a central product of quasisimple groups, which are uniquely determined. Any finite group $G$ has a unique maximal semisimple subgroup $E=E(G)$ (see [S2, Chapter 6.6]).

Let $G$ be a finite group of orientation-preserving diffeomorphisms of a homology 3 -sphere, and $E$ its maximal semisimple subgroup. If $E$ is trivial it is shown in $[\mathbf{R}$, Section 4 , case (a)] that $G$ is solvable. We will assume in the following that $E$ is nontrivial. It is shown in $[\mathbf{Z 2}]$ that the only nonabelian simple group acting on a homology 3 -sphere is the dodecahedral group $\mathbb{A}_{5}$. Now it follows from [Z1, Proposition 3] that the maximal semisimple subgroup $E$ of $G$ is of one of the following three types:

(1) $E \cong \mathbb{A}_{5}$, and every involution in $\mathbb{A}_{5}$ has nonempty connected fixedpoint set.

(2) $E \cong \mathbb{A}_{5}^{*}$, and $E$ acts freely.

(3) $E \cong \mathbb{A}_{5}^{*} \times{ }_{Z} \mathbb{A}_{5}^{*}$, and each factor $\mathbb{A}_{5}^{*}$ acts freely. 
Since $E$ is normal in $G$ its centralizer $C_{G} E$ is also normal. Let $C$ be the subgroup of $G$ generated by $E$ and $C_{G} E$. Then $C$ is normal in $G$, and the factor group $G / C$ is a subgroup of the outer automorphism group Out $E=$ Aut $E / \operatorname{Inn} E$ of $E$. The intersection of $E$ and $C_{G} E$ is the center $Z$ of $E$, which implies

$$
C \cong E \times{ }_{Z} C_{G} E
$$

Because the normalizer in $C$ of any element in $C_{G} E$ contains $E$ and hence is nonsolvable, by Propositions 1(a) and 2 the group $C_{G} E$ acts freely.

We now consider separately the three cases above.

3.1. . Suppose first that $E \cong \mathbb{A}_{5}$, and that every involution in $\mathbb{A}_{5}$ has nonempty connected fixed-point set.

Then $E$ contains a subgroup $A \cong \mathbb{Z}_{2} \times \mathbb{Z}_{2}$ (the Sylow 2-subgroup of $\mathbb{A}_{5}$ ). Let $h$ be a nontrivial involution in $A$. As all three involutions in $A$ have nonempty fixed-point set, by Proposition 1(c) the two other involutions in $A$ act as reflections (strong inversions) on the fixed-point set of $h$. The centralizer $C_{G} E$ of $E$ in $G$ is contained in the centralizer $C_{G} A$ of $A$ in $G$, which in turn is contained in the centralizer $C_{G} h$ of $h$ in $G$. Now Propositions 2 and 3 imply that $C_{G} A$, and hence also $C_{G} E$, is an elementary abelian 2 -group of rank at most three. Then $C$ is isomorphic to $\mathbb{A}_{5}$ or to $\mathbb{A}_{5} \times \mathbb{Z}_{2}$.

The outer automorphism group of $\mathbb{A}_{5}$ has order two and is generated, modulo inner automorphisms, by conjugation of $\mathbb{A}_{5}$ with any odd permutation in the symmetric group $\mathbb{S}_{5}$. Thus $C$ has index one or two in $G$. In the symmetric group $\mathbb{S}_{5}$ the equation $x y x^{-1}=y^{2}$ holds for the cycles $x=(4532)$ of order four and $y=(12345)$ of order five. Suppose that $C$ has index two in $G$. Then there is an element in $G$ that induces by conjugation the same automorphism of $\mathbb{A}_{5}$ as the cycle $x$, which contradicts Proposition 3. Thus $G=C$, and $G$ is isomorphic to $\mathbb{A}_{5}$ or $\mathbb{A}_{5} \times \mathbb{Z}_{2}$.

3.2. . Suppose that $E \cong \mathbb{A}_{5}^{*}$. Since the outer automorphism group of $\mathbb{A}_{5}^{*}$ has order two, $C \cong \mathbb{A}_{5}^{*} \times{ }_{Z} C_{G} E$ has index one or two in $G$. Representing the elements of $\mathbb{A}_{5}^{*} \cong \mathrm{SL}(2,5)$ by $2 \times 2$-matrices of determinant one, the unique nontrivial outer automorphism is given by conjugation with the matrix of order four $A:=\left(\begin{array}{ll}2 & 0 \\ 0 & 1\end{array}\right)$. The equation $A B A^{-1}=B^{2}$ holds for the matrix $B:=\left(\begin{array}{ll}1 & 1 \\ 0 & 1\end{array}\right)$ representing an element of order five in $\operatorname{SL}(2,5)$.

Supposing that $C$ has index two in $G$ we get again a contradiction to Proposition 3. Thus $G=C \cong \mathbb{A}_{5}^{*} \times{ }_{Z} C_{G} E$.

Recall that $C_{G} E$ acts freely, hence $C_{G} E$ is isomorphic to one of the groups in the list 2.1-2.6 (actually, it cannot be isomorphic to $\mathbb{A}_{5}^{*}$ because otherwise $E$ would not be the maximal semisimple subgroup of $G$; for the following arguments this makes no difference, however).

We denote by $Q_{8}=\{ \pm 1, \pm i, \pm j, \pm k\}$ the quaternion group of order eight (isomorphic to $\mathbb{D}_{8}^{*}$ ); the center $Z \cong \mathbb{Z}_{2}$ of $Q_{8}$ is generated by -1 . 
Lemma. For a positive integer $m>1$, the groups $Q_{8} \times{ }_{Z} Q_{8} \times \mathbb{Z}_{m}$ and $Q_{8} \times{ }_{Z} Q_{8} \times{ }_{Z} \mathbb{Z}_{2 m}$ do not act on a homology 3-sphere preserving orientation.

Proof. The central product $Q_{8} \times{ }_{Z} Q_{8}$ has the subgroup $\{( \pm 1,1),( \pm i, i)$, $( \pm j, j),( \pm k, k)\}$ isomorphic to $\mathbb{Z}_{2} \times \mathbb{Z}_{2} \times \mathbb{Z}_{2}$. Now the lemma follows from Proposition 3(a).

Using the lemma we shall exclude various types of groups 2.1-2.6.

(i) Suppose that $C_{G} E$ is isomorphic to a group $P_{8 \cdot 3^{k}}^{\prime}$ of type 2.3. This contains a subgroup $Q_{8} \times \mathbb{Z}_{3^{k-1}}$. The Sylow 2-subgroup of $\mathbb{A}_{5}^{*}$ is the quaternion group $Q_{8} \cong \mathbb{D}_{8}^{*}$, hence $G \cong \mathbb{A}_{5}^{*} \times{ }_{Z} C_{G} E$ contains a subgroup $Q_{8} \times{ }_{Z} Q_{8} \times \mathbb{Z}_{3^{k-1}}$. By the lemma this is possible only for $k=1$, so $C_{G} E$ is isomorphic to the binary tetrahedral group $\mathbb{A}_{4}^{*} \cong P_{24}^{\prime}$.

(ii) Suppose that $C_{G} E$ is isomorphic to a group $D_{2^{k}(2 n+1)}$ of type 2.2. Then $G \cong \mathbb{A}_{5}^{*} \times{ }_{Z} D_{2^{k}(2 n+1)}$ contains a subgroup $Q_{8} \times{ }_{Z} \mathbb{Z}_{4}$ where $\mathbb{Z}_{4}$ is generated by $u:=x^{2^{k-2}}$ (see the presentation of $D_{2^{k}(2 n+1)}$ in 2.2). Suppose $k>2$. The subgroup of $G$ containing the elements $( \pm 1,1)$ and $( \pm i, u)$ is isomorphic to $\mathbb{Z}_{2} \times \mathbb{Z}_{2}$ (note that $u^{2}$ is the unique central involution in $D_{2^{k}(2 n+1)}$ that is identified with -1 in $\left.Q_{8}\right)$. By Proposition 2, the central involution $(-1,1)=\left(1, u^{2}\right)$ in $G$ has empty fixed-point set, so by Proposition 1 we can assume that the involution $h:=(i, u)$ has nonempty connected fixed-point set. The centralizer of $h$ in $G$ contains the generators $x$ and $y$ of $D_{2^{k}(2 n+1)}$. Then both $x$ and $y$ act as rotations along the circle of fixed points of $h$ (and not as reflections, see the proof of Proposition 2). This implies that $x$ and $y$ commute, which is a contradiction. Thus $k=2$ and $D_{2^{k}(2 n+1)}=D_{4(2 n+1)}$ is isomorphic to the binary dihedral group $\mathbb{D}_{4(2 n+1)}^{*}$.

(iii) Suppose that $C_{G} E$ is isomorphic to a group $P_{48 r}^{\prime \prime}$ of type 2.5. As $C_{G} E$ acts freely, it follows from $\left[\mathbf{M n}\right.$, Lemma 2] that $r=3^{k}$, and by [L, Corollary 4.17] the group $P_{48 \cdot 3^{k}}^{\prime \prime}$ does not act freely on a homology 3 -sphere, for $k \geq 1$.

A direct argument that, for $r>1$, the group $G \cong \mathbb{A}_{5}^{*} \times{ }_{Z} P_{48 r}^{\prime \prime}$ does not act on a homology 3 -sphere is as follows: the group $P_{48 r}^{\prime \prime}$ has $\mathbb{S}_{4}^{*}$ as a quotient; the preimage of $\mathbb{A}_{4}^{*} \subset \mathbb{S}_{4}^{*}$ in $P_{48 r}^{\prime \prime}$ is a group of type 2.3 and contains a subgroup $Q_{8} \times \mathbb{Z}_{r}$ [see part (i) of the proof]. Then $G$ has a subgroup $Q_{8} \times{ }_{Z} Q_{8} \times \mathbb{Z}_{r}$ and the lemma implies $r=1$, so $C_{G} E$ is isomorphic to the binary octahedral group $\mathbb{S}_{4}^{*} \cong P_{48}^{\prime \prime}$.

By (i)-(iii), the remaining possibilities for $C_{G} E$ are the groups of type 2.1, 2.4 and the product of any of these groups with a cylic group of relatively prime order (type 2.6).

Suppose that $C_{G} E$ is a product of one of the groups $\mathbb{D}_{4 n}^{*}$ with $n$ even, $\mathbb{A}_{4}^{*}$, $\mathbb{S}_{4}^{*}, \mathbb{A}_{5}^{*}$ or $Q(8 n, k, l)$ with a cyclic group $\mathbb{Z}_{m}$. Then $C_{G} E$ has a subgroup $Q_{8} \times \mathbb{Z}_{m}$, so $G \cong \mathbb{A}_{5}^{*} \times{ }_{Z} C_{G} E$ has a subgroup $Q_{8} \times{ }_{Z} Q_{8} \times \mathbb{Z}_{m}$ and the lemma implies $m=1$. 
By [L, Corollary 4.15] the groups $Q(8 n, k, l)$ with $n$ even do not act freely on a homology 3 -sphere. This leaves for $G$ exactly the possibilities (ii), (iii) and (iv) of the theorem.

3.3. . Finally, suppose that $E \cong \mathbb{A}_{5}^{*} \times_{Z} \mathbb{A}_{5}^{*}$. Then $E$ has a subgroup $Q_{8} \times_{Z}$ $Q_{8}$. It follows from the lemma that, for $C \cong E \times{ }_{Z} C_{G} E$, the group $C_{G} E$ is equal to $Z$. Thus $C=E \cong \mathbb{A}_{5}^{*} \times{ }_{Z} \mathbb{A}_{5}^{*}$.

The outer automorphism group of $E \cong \mathbb{A}_{5}^{*} \times_{Z} \mathbb{A}_{5}^{*}$ is the dihedral group of order eight: considering centralizers of elements, each element $(x, 1)$ is mapped to an element of the form $(y, 1)$ or $(1, y)$, so each automorphism of $E$ either preserves each factor (and the subgroup generated by such automorphisms is isomorphic to $\mathbb{Z}_{2} \times \mathbb{Z}_{2}$ ) or exchanges them. It follows that $E$ has index at most eight in $G$.

It follows as in case 3.2 of the proof of the theorem that any nontrivial outer automorphism of $E$ induced by conjugation with an element of $G$ has to interchange the two factors of $E \cong \mathbb{A}_{5}^{*} \times{ }_{Z} \mathbb{A}_{5}^{*}$; in particular, $E$ has index at most two in $G$. Up to inner automorphisms, an outer automorphism $\alpha$ of order two of $\mathbb{A}_{5}^{*} \times_{Z} \mathbb{A}_{5}^{*}$ and interchanging its two factors is of the form $\alpha(x, y)=(\beta(y), \beta(x))$, for an automorphism $\beta$ of $\mathbb{A}_{5}^{*}$.

Suppose that $\alpha$ is induced by conjugation by an element $g$ in $G$. We consider the subgroup $\mathbb{A}_{5}$ of $\mathbb{A}_{5}^{*} \times{ }_{Z} \mathbb{A}_{5}^{*}$ consisting of all elements of the form $(x, x)$. If $\beta$ represents the unique nontrivial outer automorphism of $\mathbb{A}_{5}^{*}$ then conjugation by $g$ induces the unique nontrivial outer automorphism of $\mathbb{A}_{5}$. By the argument in case 3.1 of the proof of the theorem this is not possible, so we can assume $\beta$ is the trivial automorphism of $\mathbb{A}_{5}^{*}$. Then $\alpha^{2}$ is the trivial automorphism of $\mathbb{A}_{5}^{*} \times_{Z} \mathbb{A}_{5}^{*}$, and $g$ induces the trivial automorphism of $\mathbb{A}_{5}$. Proposition 2 implies that $g$ acts without fixed points (because its centralizer contains $\mathbb{A}_{5}$ ).

There are the two possibilities $g^{2}=1$ or $g^{2}=z$ (where $z$ denotes the nontrivial element of $Z$ ). If $g^{2}=1$ then the subgroup of $G$ generated by $g$ and $z$ is isomorphic to $\mathbb{Z}_{2} \times \mathbb{Z}_{2}$, and Proposition 1 implies that $g$ or $z$ has nonempty fixed-point set, which is not possible by Proposition 2. If $g^{2}=z$ then $g$ has order four and $G$ contains a subgroup $\mathbb{A}_{5} \times \mathbb{Z}_{4}$ and hence also a subgroup $\mathbb{Z}_{2} \times \mathbb{Z}_{2} \times \mathbb{Z}_{4}$, which is not possible by the lemma.

Thus $G=E$, and $g$ is isomorphic to $\mathbb{A}_{5}^{*} \times{ }_{Z} \mathbb{A}_{5}^{*}$. This finishes the proof of the theorem.

\section{References}

[AM] A. Adem and R.J. Milgram, Cohomology of Finite Groups, Grundlehren der mathematischen Wissenschaften, 309, Springer-Verlag, Berlin, 1994, MR 1317096 (96f:20082), Zbl 0820.20060.

[B] G. Bredon, Introduction to Compact Transformation Groups, Pure and Applied Mathematics, 46, Academic Press, New York, 1972, MR 0413144 (54 \#1265), Zbl 0246.57017. 
[Dr] A. Dress, Newman's theorem on transformation groups, Topology, 8 (1969), 203208, MR 0238353 (38 \#6629), Zbl 0176.53201.

[DV] P. Du Val, Homographies, Quaternions and Rotations, Oxford Mathematical Monographs Clarendon Press, Oxford, 1964, MR 0169108 (29 \#6361), Zbl 0128.15403.

[Du] W.D. Dunbar, Nonfibering spherical 3-orbifolds. Trans. Amer. Math. Soc., 341 (1994), 121-142, MR 1118824 (94c:57022), Zbl 0808.57007.

[K] R. Kirby, ed., Problems in low-dimensional topology, AMS/IP Stud. Adv. Math., 2.2, Geometric topology (Athens, GA, 1993), Amer. Math. Soc., Providence, RI, 1997, 35-473, MR 1470751.

[L] R. Lee, Semicharacteristic classes, Topology, 12 (1973), 183-199, MR 0362367 (50 \#14809), Zbl 0264.57012.

[Mg] R.J. Milgram, Evaluating the Swan finiteness obstruction for periodic groups, Algebraic and Geometric Topology (New Brunswick, N.J., 1983), Lecture Notes in Math., 1126, Springer, 1985, 127-158, MR 0802788 (87a:57048), Zbl 0572.57012.

[Mn] J. Milnor, Groups which act on $S^{n}$ without fixed points, Amer. J. Math., 79 (1957), 623-630, MR 0090056 (19,761d), Zbl 0078.16304.

[N] M.H.A. Newman, A theorem on periodic transformations of spaces, Quart. J. Math., 2 (1931), 1-9, JFM 57.0496.02.

[R] M. Reni, On finite groups acting on homology 3-spheres, J. Lond. Math. Soc., 63 (2001), 226-246, MR 1802769 (2001j:57021), Zbl 1012.57025.

[RZ] M. Reni and B. Zimmermann, On hyperelliptic involutions of hyperbolic 3manifolds, Math. Ann., 321 (2001), 295-317, MR 1893403 (2003b:57025), Zbl 0986.57012.

[S1] M. Suzuki, Group Theory I, Grundlehren der Mathematischen Wissenschaften, 247, Springer-Verlag, Berlin-New York, 1986, MR 0648772 (82k:20001c).

[S2] M. Suzuki, Group Theory II, Grundlehren der Mathematischen Wissenschaften, 248, Springer-Verlag, Berlin-New York, 1986, MR 0648772 (82k:20001c), Zbl 0586.20001.

[W] J.A. Wolf, Spaces of Constant Curvature, Publish or Perish, Boston, 1974, MR 0343214 (49 \#7958), Zbl 0281.53034.

[Z1] B. Zimmermann, Finite groups acting on homology 3-spheres: on a result of M. Reni, Monatsh. Math., 135 (2002), 253-258, MR 1897579 (2003b:57026), Zbl 0994.57019.

[Z2] B. Zimmermann, On finite simple groups acting on homology 3-spheres, Top. Appl., 125 (2002), 199-202, MR 1933571 (2003m:57041), Zbl 1012.57028.

Received January 7, 2002 and revised February 14, 2003.

Università Degli Studi di Trieste

Dipartimento di Matematica e Informatica

34100 TRIESTE

ITALY

E-mail address: zimmer@univ.trieste.it 